\title{
A Time-Series Approach to Non-Self-Financing Hedging in a Discrete-Time Incomplete Market
}

\author{
N. Josephy, L. Kimball, and V. Steblovskaya \\ Department of Mathematical Sciences, Bentley College, 175 Forest Street, Waltham, MA 02452-4705, USA
}

Correspondence should be addressed to V. Steblovskaya, vsteblovskay@bentley.edu

Received 16 May 2008; Accepted 30 July 2008

Recommended by Nikolai Leonenko

We present an algorithm producing a dynamic non-self-financing hedging strategy in an incomplete market corresponding to investor-relevant risk criterion. The optimization is a twostage process that first determines market calibrated model parameters that correspond to the market price of the option being hedged. In the second stage, an optimal set of model parameters is chosen from the market calibrated set. This choice is based on stock price simulations using a time-series model for stock price jump evolution. Results are presented for options traded on the New York Stock Exchange.

Copyright $@ 2008$ N. Josephy et al. This is an open access article distributed under the Creative Commons Attribution License, which permits unrestricted use, distribution, and reproduction in any medium, provided the original work is properly cited.

\section{Introduction}

This paper is a continuation of the work originally presented in [1] where we developed an algorithm producing a dynamic non-self-financing hedging strategy in an incomplete market optimizing a suitable investor-relevant risk criterion. The algorithm expanded on theoretical investigations of A. V. Nagaev et al. (see [2-5]) where the authors studied asymptotic behavior of the residual value of a minimum cost super-hedge. The residual value occurs as a result of a non-self-financing dynamic hedging strategy of an option seller in an incomplete market introduced and discussed in [2].

The financial market model considered by Nagaev et al. consists of a bond, a stock, and a European type derivative security with a convex payoff function. The stock price jumps are assumed to be distributed over a bounded interval. This model is a natural extension of the classical binomial model of Cox-Ross-Rubinstein as well as its further multinomial extensions (see, e.g., [6-8], and references therein).

The model considered in this paper as well as its multinomial predecessors produces an incomplete market. A significant proportion of research on option pricing and hedging in incomplete markets constructs self-financing trading strategies that satisfy both a primary noarbitrage condition and secondary conditions on portfolio risk and return. A comprehensive 
survey of modern methodologies can be found in [9]. A number of articles that deal with frictions in markets, shortfall risks, and quadratic hedging (all producing incomplete markets) can be found in the recent compendium [10].

Less prevalent is the study of non-self-financing trading strategies in similar economic environments. The encyclopedic reference [11] and the more modest [12] both illuminate option pricing with consumption, the model which is similar to the work presented here. The application of constrained quadratic programming to the pricing of options by constructing non-self-financing portfolios in an incomplete market can be found in [13]. We note that the alternative approach of specifying a deterministic model of incomplete markets is an active area of research, as illustrated by [14-17].

In [1] as well as in the present work, we study the short-term behavior of the residual value of a dynamic non-self-financing hedging strategy, whose long-term behavior was studied by Nagaev in [5]. Our goal is to develop and evaluate an algorithm that will determine a dynamic non-self-financing hedging strategy consisting of a portfolio of our stock and bond assets in an incomplete market. The portfolio will approximately hedge the derivative security and will satisfy additional criteria that are meaningful to the investor, based on the deviation of the portfolio value from the required hedging value.

Our algorithm is a two-stage process that first determines a set of market calibrated model parameters that correspond to the market price of the option being hedged. In the second stage, an optimal set of model parameters is chosen from this set. This choice is based on stock price simulations. In our initial work [1], we used a bootstrap method to simulate future stock price paths assuming that the stock price jumps are independent identical distributed random variables. In the present work, we relax the i.i.d. assumption on the stock price jumps and use a time-series model for stock price jump evolution.

We fit an ARMA model to historical stock price jump series and extract the correlation structure in the stock price jump process. Further, we simulate ARMA model driving noise to construct future values of the price jump process by using the nonparametric bootstrap method of sampling with replacement from the ARMA model residual sequence. The above method (unlike common parametric methods based on an assumed Gaussian distribution) captures long tails observed in typical stock price jump distributions.

The remainder of the paper is organized as follows. We develop the discrete time financial model in Section 2. The notion of the residual value of a minimum cost superhedge is described in Section 3. Non-self-financing dynamic hedging strategies and residual values associated with them are discussed in Section 4 . Section 5 contains a brief overview of our hedging algorithm. The first stage of the algorithm, where we find the set of market calibrated model parameters, is presented in Section 6. Section 7 describes the second stage of the algorithm: the numerical optimization over the set of market calibrated model parameters in order to determine an optimal hedging strategy based on a risk criterion chosen by an investor. Section 8 is devoted to the ARMA model based stock price simulation. The results of applying our algorithm to a variety of stock options are presented in Section 9 . Section 10 presents the comparison of our optimal hedging strategy with the Black-Scholes hedging strategy. We conclude with some remarks in Section 11.

\section{Discrete-time market model and no-arbitrage option prices}

Our discrete-time financial market model consists of two fundamental assets and a derivative security. 
(1) A risk-free bond with fixed periodic interest rate $r$, evolving from an initial value $b_{0}>0$ at time $t=0$ to $b_{k}$ at time $t=k$ as

$$
b_{k}=b_{0}(1+r)^{k}
$$

(2) A risky stock evolving from an initial value $s_{0}$ at time $t=0$ to $s_{k}$ at time $t=k$ as

$$
s_{k}=s_{0} \xi_{1} \xi_{2} \cdots \xi_{k}
$$

where the stock price jumps $\xi_{k}=s_{k} / s_{k-1}$ are assumed to be random variables distributed over a bounded interval $[D, U], D<U$ at every time step $k$. No further assumptions are made on the distribution for the $\xi_{k}$.

(3) A European type option with expiry $n$ and convex payoff function $f$.

We will require the condition

$$
D<1+r<U
$$

to be fulfilled to guarantee no-arbitrage in this market.

This discrete-time market model is incomplete. Indeed it is well known that the famous binomial model is complete while its trinomial (as well as more general multinomial) extensions are not (see, e.g., [6]). Our model generalizes the multinomial models allowing the stock price jumps $\xi_{k}$ at every time step $k$ to be distributed over a bounded interval $[D, U], D<U$. The incompleteness of the model is manifested in an open interval $\left(\underline{x}_{k}, \bar{X}_{k}\right)$ $(k=0, \ldots, n-1)$ of no-arbitrage option prices (see, e.g., $[2,18])$. It is shown in [18] that the end points of the interval are given by the following formulas:

$$
\begin{aligned}
\bar{X}_{k}(U, D) & =g_{k}\left(U, D, s_{k}\right), \\
\underline{x}_{k}(U, D) & =(1+r)^{-(n-k)} f\left(s_{k}(1+r)^{n-k}\right),
\end{aligned}
$$

where $D$ and $U$ define the support of the stock price jump distribution (see above), $f$ is a convex payoff function of the option, $n$ is the number of periods to the option expiration, $s_{k}$ is the stock price at time $k$, and $g_{k}$ is defined as follows:

$$
\begin{gathered}
g_{k}(U, D, s)=(1+r)^{-(n-k)} \sum_{j=0}^{n-k}\left(\begin{array}{c}
n-k \\
j
\end{array}\right)[p(U, D)]^{j}[1-p(U, D)]^{n-k-j} f\left(s U^{j} D^{n-k-j}\right), \\
p(U, D)=\frac{(1+r)-D}{U-D} .
\end{gathered}
$$

(Here, $\left(\begin{array}{c}n-k \\ j\end{array}\right)$ is the binomial coefficient.)

For the option seller, the upper bound $\bar{X}_{k}(U, D)$ is the demarcation between risk sharing with the option buyer (if the option sale price is below $\left.\bar{X}_{k}(U, D)\right)$ and the potential for arbitrage profit (if the option sale price is at or above $\bar{X}_{k}(U, D)$ ). 


\section{Minimum cost super hedge}

For the rest of the paper, we take the position of an option seller who wishes to hedge the potential liability of the sold option being exercised. In this section, we will consider an extreme case: suppose the option with the convex payoff function $f$ is sold at time $t=0$ for the upper bound price $\bar{X}_{0}(U, D)$. The option seller uses the amount $\bar{X}_{0}(U, D)$ to finance the setup cost of a portfolio consisting of $\gamma_{0}$ stocks and $\beta_{0}$ bonds with the intention of hedging the short position in the option:

$$
\bar{X}_{0}(U, D)=\gamma_{0} s_{0}+\beta_{0} b_{0} .
$$

The seller rebalances the portfolio at each time instant $t=k(k=1, \ldots, n-1)$ creating a dynamic trading strategy $\left(\gamma_{k}, \beta_{k}\right)(k=0, \ldots, n-1)$.

We will choose the quantities $\gamma_{k}$ and $\beta_{k}$ according to the formulas

$$
\begin{aligned}
& \gamma_{k}=\gamma_{k}(U, D)=\frac{g_{k+1}\left(U, D, s_{k} U\right)-g_{k+1}\left(U, D, s_{k} D\right)}{s_{k}(U-D)}, \\
& \beta_{k}=\beta_{k}(U, D)=\frac{U g_{k+1}\left(U, D, s_{k} D\right)-D g_{k+1}\left(U, D, s_{k} U\right)}{(1+r) b_{k}(U-D)},
\end{aligned}
$$

where $g_{k}$ is defined in (2.6). S. A. Nagaev and A. V. Nagaev [2] showed (based on a convexity argument) that the dynamic trading strategy $\left(\gamma_{k}, \beta_{k}\right)(k=0, \ldots, n-1)$ represents a minimum cost super-hedging strategy in the following sense: the associated portfolio value at every time instant $t=k$ is greater than or equal to the value of the option. It is worth explaining the above statement in more detail.

Suppose at each time instant $k$, the option seller liquidates the portfolio constructed in the prior period $[k-1, k)$ and uses the proceeds to construct a new portfolio for the current period $[k, k+1)$. Let us denote by $v_{k}(U, D)$ the liquidation value of the prior period portfolio:

$$
\begin{aligned}
v_{k}(U, D) & =\gamma_{k-1}(U, D) s_{k}+\beta_{k-1}(U, D) b_{k} \\
& =\gamma_{k-1}(U, D) s_{k-1} \xi_{k}+\beta_{k-1}(U, D) b_{k-1}(1+r) .
\end{aligned}
$$

Combining (3.4) with (3.2) and (3.3), one gets

$$
v_{k}(U, D)=\frac{U-\xi_{k}}{U-D} g_{k}\left(U, D, s_{k-1} D\right)+\frac{\xi_{k}-D}{U-D} g_{k}\left(U, D, s_{k-1} U\right) .
$$

On the other hand, the funds required to finance the current period portfolio, or setup cost, are given by the upper bound $\bar{X}_{k}(U, D)$ of the no-arbitrage price interval corresponding to the time moment $t=k$ :

$$
\bar{X}_{k}(U, D)=g_{k}\left(U, D, s_{k-1} \xi_{k}\right)
$$

(see (2.4)). The difference between the liquidation value (3.5) and the setup cost (3.6) is a residual amount $\delta_{k}$ :

$$
\delta_{k}(U, D)=\frac{U-\xi_{k}}{U-D} g_{k}\left(U, D, s_{k-1} D\right)+\frac{\xi_{k}-D}{U-D} g_{k}\left(U, D, s_{k-1} U\right)-g_{k}\left(U, D, s_{k-1} \xi_{k}\right) .
$$


Since we assume that $D \leq \xi_{k} \leq U$, it follows from the convexity of the payoff function $f$ that the residual is nonnegative:

$$
\delta_{k}(U, D) \geq 0, \quad k=1, \ldots, n .
$$

In this fashion, at every time step $k$, the option seller obtains a nonnegative residual $\delta_{k}(U, D)$, which is withdrawn after each portfolio liquidation prior to the construction of the next time period super-hedge. The above constructed super-hedging strategy is in general non-self-financing.

The accumulated value of the withdrawn residuals at maturity, which we will refer to as the minimum cost super-hedge residual, is given by

$$
\Delta_{n}(U, D)=\delta_{1}(U, D)(1+r)^{n-1}+\delta_{2}(U, D)(1+r)^{n-2}+\cdots+\delta_{n}(U, D) .
$$

\section{Non-self-financing hedging strategies and their residuals}

In the previous section, we considered a hypothetical situation where the option has been sold at time $t=0$ for the price equal to the upper bound $\bar{X}_{0}(U, D)$ of the no-arbitrage option price interval. We saw how the option seller could use this option premium to construct a minimum cost super hedge based on a non-self-financing strategy.

In this section, we consider a more realistic situation where the initial (time zero) option price is lower than the upper bound $\bar{X}_{0}(U, D)$, but still falls within the open interval of no-arbitrage option prices $\left(\underline{x}_{0}(U, D), \bar{X}_{0}(U, D)\right)$. In this case, the option seller cannot in general construct a super hedge, but it is possible to construct a non-self-financing trading strategy that will produce a possibly nonnegative residual amount.

In order to explain how such trading strategy can be constructed, we need to present the following short mathematical introduction. Let us consider the quantity $x_{k}(u, d)$ given as follows:

$$
x_{k}(u, d)=g_{k}\left(u, d, s_{k}\right), \quad k=0, \ldots, n-1,
$$

where $s_{k}$ is the stock price at time $k$, and $g_{k}$ is defined by (2.6) with boundary parameters $U$, $D$ replaced with the values $u, d$ such that

$$
D \leq d \leq 1+r \leq u \leq U .
$$

The next proposition follows from the convexity arguments.

Proposition 4.1. Let $f$ be a convex function. The function $x_{k}(u, d)$ maps the set of $(u, d)$ pairs satisfying (4.2) onto the option price interval $\left[\underline{x}_{k}(U, D), \bar{X}_{k}(U, D)\right]$ defined in (2.4) and (2.5). When $(u, d)=(U, D), x_{k}(u, d)=\bar{X}_{k}(U, D)$ and when $(u, d)=(1+r, 1+r)$, then $x_{k}(u, d)=\underline{x}_{k}(U, D)$.

The above proposition infers that for any choice of $d$ and $u$ satisfying

$$
D<d<1+r<u<U,
$$

the quantity (4.1) falls within the no-arbitrage option price interval

$$
\underline{x}_{k}(U, D)<x_{k}(u, d)<\bar{X}_{k}(U, D), \quad k=0, \ldots, n-1 .
$$


Conversely, every no-arbitrage market option price $y$ (every point $y \in\left(\underline{x}_{k}(U, D), \bar{X}_{k}(U, D)\right)$ ) can be put into correspondence with at least one pair $(u, d)$ satisfying $(4.3): y=x_{k}(u, d)$.

Suppose the option with the convex payoff function $f$ was sold at time $k=0$ for the price $\bar{x}_{0}$. If we assume that there is no arbitrage on the market, then there is at least one $(u, d)$ pair satisfying (4.3) that allows the amount $\bar{x}_{0}$ to be identified with the no-arbitrage option price $x_{0}(u, d)$ satisfying $(4.4)$ with $k=0$. In other words,

$$
\bar{x}_{0}=x_{0}(u, d) .
$$

We remark here that the exact values of the boundary parameters $D$ and $U$ are not important for practical purposes of option hedging, since they play purely theoretical role in our setting.

Let us return to (4.5). There are an infinite number of $(u, d)$ pairs satisfying (4.5). For a fixed observed market option price $\bar{x}_{0}$, we will call the set of $(u, d)$ pairs satisfying $(4.5)$ the market calibrated set of model parameters. Our goal will be to choose the best market calibrated $(u, d)$ pair based on an optimization criterion explained in Section 7.

The option seller uses the amount $\bar{x}_{0}$ to setup the hedging portfolio $\left(\gamma_{0}(u, d), \beta_{0}(u, d)\right)$ :

$$
\bar{x}_{0}=\gamma_{0}(u, d) s_{0}+\beta_{0}(u, d) b_{0},
$$

which will be rebalanced at every time instant $t=k$ following the dynamic portfolio strategy

$$
\left(\gamma_{k}(u, d), \beta_{k}(u, d)\right), \quad k=0, \ldots, n-1,
$$

where $\gamma_{k}(u, d)$ and $\beta_{k}(u, d)$ are defined in (3.2) and (3.3), respectively, with the boundary parameters $U, D$ replaced with the values $u, d$ :

$$
\begin{aligned}
& \gamma_{k}(u, d)=\frac{g_{k+1}\left(u, d, s_{k} u\right)-g_{k+1}\left(u, d, s_{k} d\right)}{s_{k}(u-d)}, \\
& \beta_{k}(u, d)=\frac{u g_{k+1}\left(u, d, s_{k} d\right)-d g_{k+1}\left(u, d, s_{k} u\right)}{(1+r) b_{k}(u-d)} .
\end{aligned}
$$

We remark that in fact there is an infinite number of dynamic portfolio strategies (4.7) defined by the formulas (4.8). These strategies are distinguished by the values of the market calibrated parameters $(u, d)$. We will call them the market calibrated dynamic portfolio strategies, or simply market calibrated hedging strategies.

By analogy with the arguments of Section 3 , for every $k=1, \ldots, n$, one can calculate the difference between the liquidation value of the portfolio constructed in the period $[k-$ $1, k)$ and the setup cost of the portfolio constructed for the period $[k, k+1)$. This difference constitutes a residual value

$$
\delta_{k}(u, d)=\frac{u-\xi_{k}}{u-d} g_{k}\left(u, d, s_{k-1} d\right)+\frac{\xi_{k}-d}{u-d} g_{k}\left(u, d, s_{k-1} u\right)-g_{k}\left(u, d, s_{k-1} \xi_{k}\right) .
$$

The latter formula (4.9) was obtained by analogy with the formula (3.7), which represents the residual amount corresponding to the boundary case of the minimum cost super hedge.

It is straightforward to show that

(i) $\delta_{k}(u, d)>0$, if $d<\xi_{k}<u$;

(ii) $\delta_{k}(u, d)=0$, if $\xi_{k}=d$ or $\xi_{k}=u$;

(iii) $\delta_{k}(u, d)<0$, if $D<\xi_{k}<d$ or $u<\xi_{k}<U$. 
In order to maintain the dynamic portfolio strategy defined by (4.7), at each time step $k=$ $1, \ldots, n$, the investor will either withdraw the residual (4.9) from the liquidated proceeds when $\delta_{k}(u, d)>0$ or add the amount when $\delta_{k}(u, d)<0$. The local residuals $\delta_{k}(u, d)$ produce an accumulated residual at option expiration:

$$
\Delta_{n}(u, d)=\delta_{1}(u, d)(1+r)^{n-1}+\delta_{2}(u, d)(1+r)^{n-2}+\cdots+\delta_{n}(u, d) .
$$

Note that the accumulated residual defined in (4.10) differs from the minimum cost superhedge residual defined in (3.9) as illustrated by the characterizations of $\delta_{k}$ given above. S.A. Nagaev and A. V. Nagaev studied asymptotic properties of the minimum cost super-hedge residual (3.9) (or riskless profit of the investor, in his terminology) extensively in [2-4]. That work was extended to the asymptotic properties of the accumulated residual (4.10) in [5]. Our work investigates the short-term behavior of the accumulated residual and its usefulness in constructing practical hedging strategies for this market model.

Remark 4.2. We would like to stress here that the dynamic portfolio strategy constructed in (4.7) is in general non-self-financing. Also, although the strategy provides an approximate hedging of the short position in an option, for the sake of simplicity we will still call it a hedging strategy.

Given the market option price $\bar{x}_{0}$, theoretically one has an infinite choice of market calibrated model parameters $((u, d)$ pairs $)$ each determining a market calibrated hedging strategy. Each market calibrated hedging strategy produces a residual sequence. An investor (an option seller) will want to choose values for $d$ and $u$ that determine a residual sequence with additional desirable risk/return characteristics. It is the choice of the model parameter values $d$ and $u$ based on the risk/return characteristics of the residual sequence that constitutes our algorithm design.

For the remainder of this paper, we will assume a European call option payoff function $f$ :

$$
f(s)=(s-K)_{+}
$$

where $K$ is the option strike price. Also, for all our computations we will use the value $r=0$ for the risk-free interest rate. The latter is justified by the results of our computational experiments confirming that a realistic variation in values of $r$ has a minimal effect on the results for the case of short-term option contracts studied in this paper.

\section{Brief overview of the hedging algorithm}

We will start with the brief summary of the previous sections. As was explained in Section 2, the incompleteness of our market model results in an infinite number of possible no-arbitrage option prices located within the open interval $\left(\underline{x}_{0}(U, D), \bar{X}_{0}(U, D)\right)$. Assuming that there is no arbitrage on the market, we can associate a market option price $\bar{x}_{0}$ with one of the points within the theoretical interval of no-arbitrage option prices: $\bar{x}_{0}=x_{0}(u, d) \in$ $\left(\underline{x}_{0}(U, D), \bar{X}_{0}(U, D)\right)$. The infinite set of $(u, d)$ pairs for which the equality $\bar{x}_{0}=x_{0}(u, d)$ holds is called a market calibrated set of model parameters.

Each no-arbitrage option price $x_{0}(u, d)$ gives rise to a non-self-financing dynamic hedging strategy $\left(\gamma_{k}(u, d), \beta_{k}(u, d)\right), k=0, \ldots, n-1$, that is distinguished by the value of the parameters $(u, d)$. If the no-arbitrage option price $x_{0}(u, d)$ under consideration is identified 


\begin{tabular}{|c|c|c|c|c|}
\hline \multirow{2}{*}{$\begin{array}{l}\text { Historical stock prices } \\
\text { Current stock price } \\
\text { Strike price } \\
\text { Current option price }\end{array}$} & Stage 1 & \multirow{2}{*}{$\rightarrow \begin{array}{l}\text { Market calibrated set } \\
\text { of model parameters } \\
((u, d) \text { pairs })\end{array}$} & \multirow[t]{2}{*}{ Stage 2} & \multirow{2}{*}{$\begin{array}{l}\text { Optimal }\left(u^{*}, d^{*}\right) \text { pair } \\
\text { determining the optimal } \\
\text { hedging strategy } \\
\left(\gamma_{k}\left(u^{*}, d^{*}\right), \beta_{k}\left(u^{*}, d^{*}\right)\right)\end{array}$} \\
\hline & & & & \\
\hline
\end{tabular}

Figure 1: Two-stage algorithm.

with the actual market option price $\bar{x}_{0}$ so that $\bar{x}_{0}=x_{0}(u, d)$, we will call the associated hedging strategies $\left(\gamma_{k}(u, d), \beta_{k}(u, d)\right)$ market calibrated.

Each market calibrated hedging strategy $\left(\gamma_{k}(u, d), \beta_{k}(u, d)\right)$ produces a residual sequence and an accumulated residual amount $\Delta_{n}(u, d)$, when applied to a given stock price path. By imposing a risk criterion on the residual sequence, we can choose a unique optimal pair of parameters $\left(u^{*}, d^{*}\right)$ by optimizing the risk criterion over the set of market calibrated parameters. This results in the choice of the corresponding optimal market calibrated hedging strategy $\left(\gamma_{k}\left(u^{*}, d^{*}\right), \beta_{k}\left(u^{*}, d^{*}\right)\right)$.

This optimization is accomplished computationally by selecting a representative finite subset from the infinite set of market calibrated model parameters $((u, d)$ pairs $)$ and simulating a large number of stock price paths for each selected $(u, d)$ pair. Each stock price path determines a residual sequence, from which we can estimate a criterion value using a suitable sample statistic.

Figure 1 gives a brief scheme of our proposed two-stage algorithm.

We will now proceed with a more detailed explanation of each stage of the algorithm.

\section{Market calibrated set of the model parameters: stage 1 of the algorithm}

Let us place ourselves in the setting described in Section 4. At time $t=0$, the option seller receives a premium $\bar{x}_{0}$, which he/she is willing to use in order to build a dynamic hedging strategy that depends on the unknown parameters $u$ and $d$. Our goal at the first stage of the algorithm is to choose a set of market calibrated parameters $u$ and $d$. In order to do so we will identify the market option premium $\bar{x}_{0}$ with a no-arbitrage option price $x_{0}(u, d)$ :

$$
\bar{x}_{0}=x_{0}(u, d)=g_{0}\left(u, d, s_{0}\right) \text {, }
$$

(see $(4.1)$ ). The market calibrated parameters $(u, d)$ must satisfy $(6.1)$, where $g_{0}\left(u, d, s_{0}\right)$ is given by

$$
\begin{gathered}
g_{0}\left(u, d, s_{0}\right)=(1+r)^{-n} \sum_{j=0}^{n}\left(\begin{array}{l}
n \\
j
\end{array}\right)[p(u, d)]^{j}[1-p(u, d)]^{n-j}\left(s_{0} u^{j} d^{n-j}-K\right)_{+} \\
p(u, d)=\frac{(1+r)-d}{u-d} .
\end{gathered}
$$

It is more convenient to deal with the normalized quantity $g_{0}\left(u, d, s_{0}\right) / s_{0}$ which we will denote by $c_{0}(u, d)$

$$
c_{0}(u, d)=\frac{g_{0}\left(u, d, s_{0}\right)}{s_{0}}=(1+r)^{-n} \sum_{j=0}^{n}\left(\begin{array}{l}
n \\
j
\end{array}\right)[p(u, d)]^{j}[1-p(u, d)]^{n-j}\left(u^{j} d^{n-j}-R\right)_{+},
$$




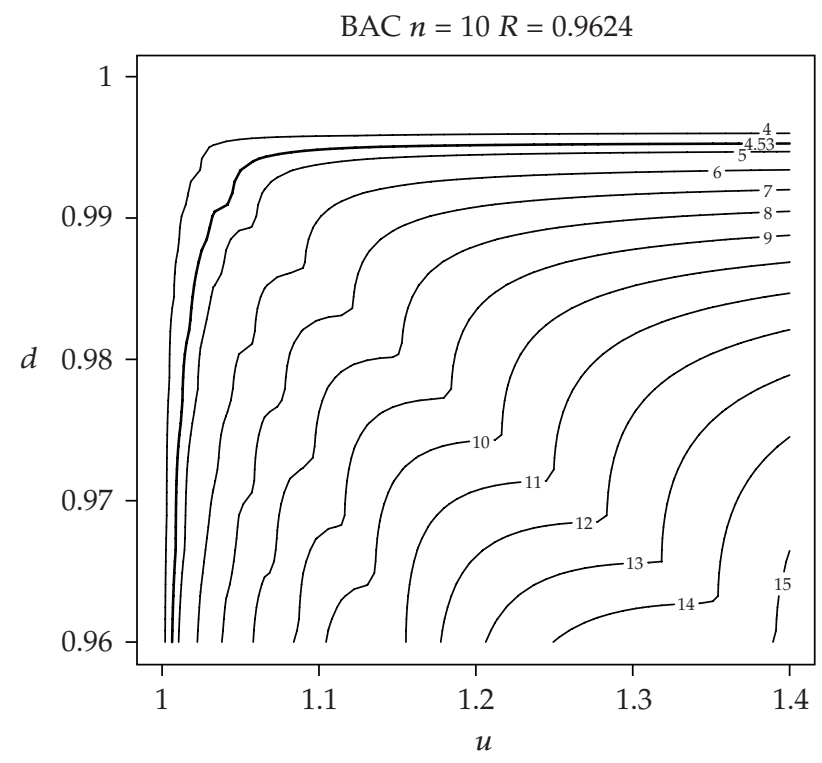

Figure 2: Contours for BAC+JV option.

where

$$
R=\frac{K}{S_{0}} .
$$

We will be interested in the level curves $\Sigma$ of the surface $c_{0}(u, d)$ (we will call them contours):

$$
\Sigma_{c^{*}}=\left\{(u, d): c_{0}(u, d)=c^{*}\right\} .
$$

The set of market calibrated model parameters $((u, d)$ pairs) is represented by the contour with

$$
c^{*}=\frac{\bar{x}_{0}}{s_{0}} .
$$

We recall that $\bar{x}_{0}$ is the market option price at time $t=0$, and $s_{0}$ is the time zero stock price.

Several contours for a representative option are shown in Figure 2. The option BAC+JV is a Bank of America call option expiring on October 15, 2004 with a strike price of $\$ 42.50$ and a current stock price of $\$ 44.16$. The contours shown each correspond to a value of $c^{*}$ (indicated on the curve scaled by a factor of 100), and the contour shown in bold is the set of market calibrated model parameters described by (6.5) and (6.6) with a value of $c^{*}=.0453$. The value of $R=0.9624$ (being less than 1) indicates that the call option is currently in the money. The shape of the market calibrated contour is similar for all data sets examined.

For the sake of comparison, Figure 3 depicts the set of contours for an Intel call option (INTC+JE) that is out of the money $(R=1.199)$. The option expired October 15, 2004 with a strike price of $\$ 25$. The market calibrated contour (indicated in bold) corresponds to the value $c^{*}=0.0024$. The shape of the contour differs slightly from the market calibrated contour shown in Figure 2. The shape of this contour is similar for other out of the money options.

The first stage of our algorithm is accomplished computationally by utilizing contour construction software to compute a finite number of market calibrated $(u, d)$ pairs satisfying (6.5) and (6.6). There are typically between 90 and $100(u, d)$ pairs identified on the market calibrated contour. It is this set of $(u, d)$ pairs that is used by the second stage of our algorithm. 


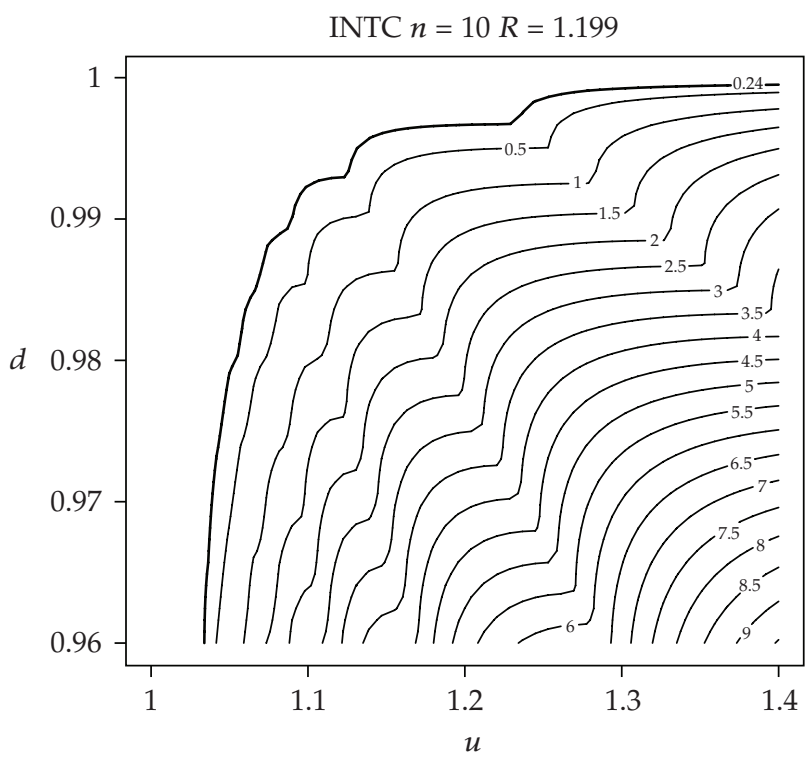

Figure 3: Contours for INTC+JE option.

\section{Choosing the optimal hedging strategy: stage 2 of the algorithm}

Let us recall that each $(u, d)$ pair on the market calibrated contour $\Sigma$ defined in (6.5) and (6.6) determines a market calibrated dynamic hedging strategy $\left(\gamma_{k}(u, d), \beta_{k}(u, d)\right), k=0, \ldots, n-1$. For a given stock price path $\left\{s_{k}\right\}$, this hedging strategy produces a sequence of residuals $\delta_{k}(u, d), k=1, \ldots, n$, each representing a residual profit/loss for an investor. This sequence is the economic consequence of choosing model parameters $(u, d)$ and the associated dynamic hedging portfolio.

The second stage of the two-stage algorithm selects a unique $(u, d)$ pair on the market calibrated contour $\Sigma$. This chosen pair of parameters $\left(u^{*}, d^{*}\right)$ uniquely defines a hedging strategy $\left(\gamma_{k}\left(u^{*}, d^{*}\right), \beta_{k}\left(u^{*}, d^{*}\right)\right), k=0, \ldots, n-1$, that will numerically optimize a risk criterion chosen by the investor.

We consider three possible criteria to choose from. These criteria convert the residual sequence into a scalar measure of investor risk, each reflecting some aspect of the option seller attitude towards risk.

(i) Maximize the likelihood of a positive accumulated residual:

$$
\max _{(u, d) \in \Sigma} \operatorname{prob}\left(\Delta_{n}(u, d)>0\right) .
$$

(ii) Minimize expected shortfall:

$$
\min _{(u, d) \in \Sigma} E(\text { short fall })=\min _{(u, d) \in \Sigma} E\left(\max _{1 \leq k \leq n}\left(-\delta_{k}(u, d)\right)\right) .
$$

(iii) Maximize the expected accumulated profit:

$$
\max _{(u, d) \in \Sigma} E\left(\Delta_{n}(u, d)\right) .
$$


The first criterion (7.1) interprets a positive residual as a profit and chooses a $(u, d)$ pair that has the highest probability of a net profit. In the absence of arbitrage, a large accumulated profit is not attainable with high probability. There is the possibility, however, of an investor achieving a small positive profit. The optimization problem presented here produces a market calibrated hedging strategy that maximizes the likelihood of a positive accumulated profit.

The second criterion (7.2) reflects an investor's desire to minimize the amount of single period additional funding needed to rebalance the portfolio over the life of the option. A negative residual $\delta_{k}(u, d)$ represents the cash shortfall in rebalancing the portfolio at time $k$. The largest negative $\delta_{k}(u, d)$ is the largest shortfall value. Optimizing this criterion produces a hedging portfolio with minimal expected single period additional funding.

Our final criterion (7.3) maximizes the expected accumulated residual, which reflects total net profit from using the dynamic portfolio strategy based on the chosen $(u, d)$. It was shown in [2] that the expected accumulated profit is asymptotically constant on contours of constant no-arbitrage price. We thus anticipate minimal differences in the expected accumulated profit at each $(u, d)$ pair on our market calibrated contours when $n$ is large. For small $n$, empirical results show that it is possible to have a market contour with nonconstant expected accumulated profit.

Let us choose one of the listed criteria. The process of selecting a unique optimal pair of parameters consists of the following. For each $(u, d)$ pair in the market calibrated subset, we simulate a number of stock price time series $\left\{s_{k}\right\}, k=1, \ldots, n$. We then apply the hedging strategy associated with the given $(u, d)$ pair to each of the simulated paths in order to determine a sequence of residuals $\left\{\delta_{k}(u, d), k=1, \ldots, n\right\}$. A particular choice of a risk criterion reduces the sequence $\left\{\delta_{k}(u, d)\right\}$ to a single scalar value of risk (described in (7.1) through (7.3)). For example, the appropriate scalar value of risk for the criterion (7.3) is the accumulated residual $\Delta_{n}(u, d)$. For a given $(u, d)$ pair, we collect the corresponding sample of scalar risk values (the sample size equals the number of the simulated stock price paths). An appropriate sample statistic (mean value or probability of a desirable event) is then computed from the sample as the utility value of the $(u, d)$ pair. The best value of the sample statistic, as the $(u, d)$ pair is varied over the market calibrated contour, is chosen as the optimal $(u, d)$. For example, the optimal $(u, d)$ pair for criterion (7.3) will correspond to the largest averaged accumulated residual $\Delta_{n}(u, d)$.

A description of the modeling process for the stock price time series is described in the following section.

\section{Stock price process modeling}

In our initial study (see [1]), we examined hedging portfolios produced by a risk minimization algorithm that simulated a stock price process under the assumption of independence of stock price jumps. A bootstrap procedure was used to sample stock price jumps with replacement from historical data to generate stock price paths. This approach assumed the independence of the sample stock price jumps. In this paper, we consider a more sophisticated model of the stock price process. We use time-series model to extract the correlation structure in the stock price jump process, producing a more structured model of the stock price process that accounts for autocorrelation in the stock price jump data.

Time-series models are developed for stock price jumps $\xi_{k}$. We fit an ARMA $(p, q)$ model of the form

$$
\xi_{k}=\alpha_{1} \xi_{k-1}+\alpha_{2} \xi_{k-2}+\cdots+\alpha_{p} \xi_{k-p}+\theta_{1} \epsilon_{k-1}+\theta_{2} \epsilon_{k-2}+\cdots+\theta_{q} \epsilon_{k-q}+\epsilon_{k} \quad k=1, \ldots, n
$$




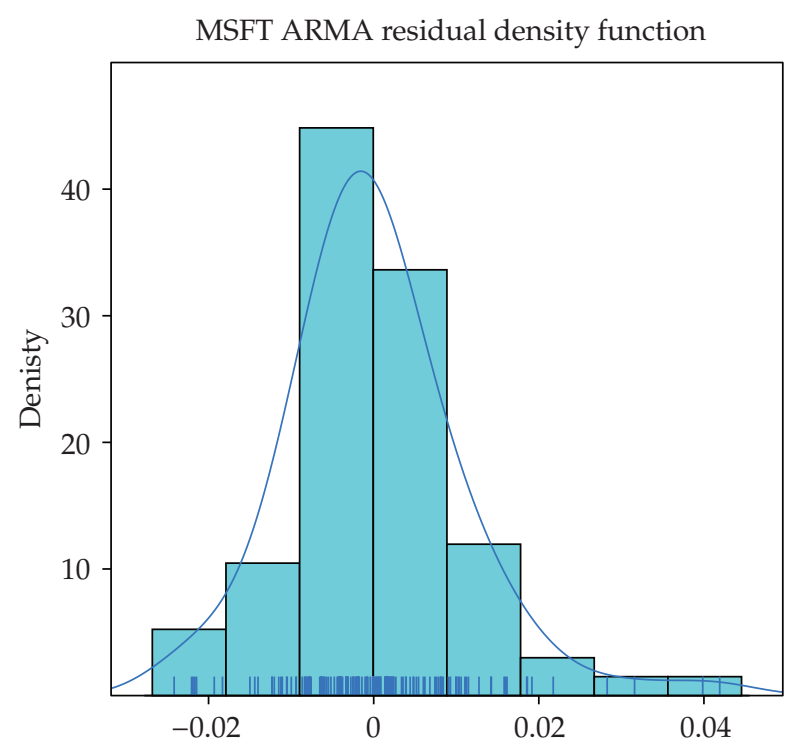

Figure 4: ARMA residual density function.

to historical price jump series. Here, $\alpha_{1}, \ldots, \alpha_{p}$ and $\theta_{1}, \ldots, \theta_{q}$ are the parameters of the model, while $\epsilon_{k}, \epsilon_{k-1}, \ldots, \epsilon_{k-q}$ are the error terms. Examining the data for the stock price jumps, it is apparent that the jump series is stationary having approximately constant mean and variance. It is also important to note that we are dealing with short-term behavior, so long-term trends and seasonality are not an issue.

The appropriate model order is determined individually for each data set based on the standard analysis of several factors (see [19]). A preliminary estimate of $(p, q)$ is made by examining the autocorrelation $(\mathrm{ACF})$ and partial autocorrelation (PACF) functions. The model fit for several values of $(p, q)$ is evaluated using diagnostic statistics. The evaluation criteria include the statistical significance level of individual ARMA terms, Akaike information criterion (AIC) value associated with the model, and validation of the noise assumptions. The lack of correlation in the residual noise sequence is established using the Box-Ljung statistics. Although many of the data sets considered indicate a $p=1, q=1$ model, each data set exhibits unique patterns, and higher order models are appropriate in some cases. The model parameters are evaluated based on the model order. The time-series model for the stock price jump process and an appropriate residual noise process can be used to simulate future values of the stock price jumps (and then stock prices).

Common parametric methods for generating residual noise values include random number generation using an assumed underlying distribution. Examining the residuals in our time-series jump data, we see that the distribution has a long tail indicative of a nonnormal distribution (see Figure 4). To guarantee the appropriate inclusion of such tail valued price jumps in our simulation, the method chosen for simulating ARMA model driving noise for future values of the price jump process is the nonparametric bootstrap method of sampling with replacement from the residual sequence $\left\{\epsilon_{k}\right\}$.

Using the ARMA residual sequence as noise, we simulate future values of the stock jump process. The jump process is accumulated to form a stock price process, which can be used to calculate a residual sequence $\delta_{k}$ or an accumulated residual $\Delta_{n}$ using our risk minimization algorithm. This process is summarized in the flow chart shown in Figure 5. 


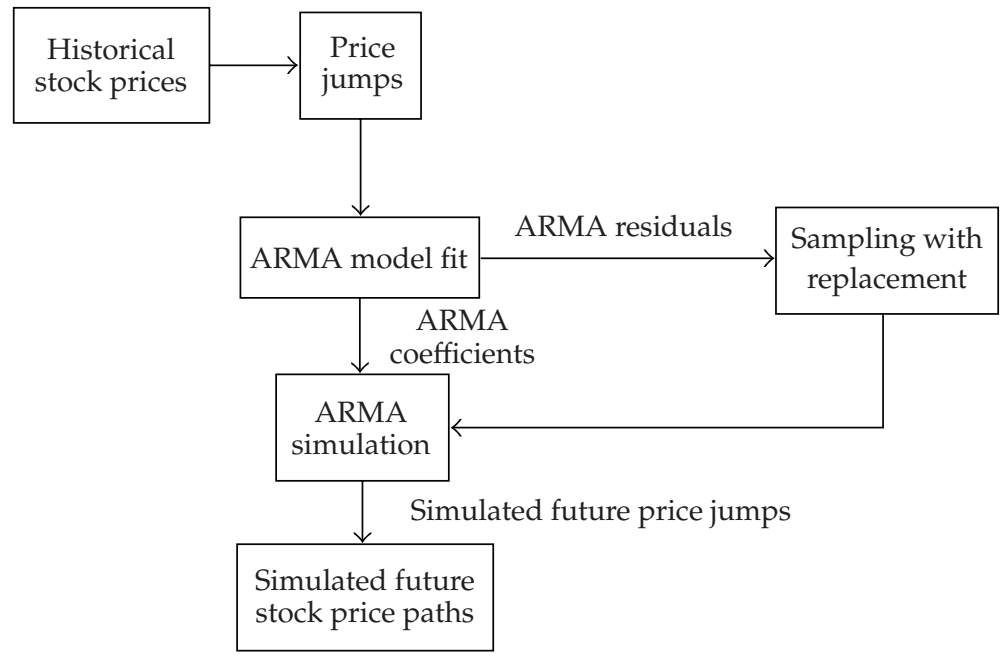

Figure 5: Simulation of stock price paths.

We conclude this section with a brief overview of the existing literature on autoregressive processes with given marginal distributions. In our model, we assume that the stock price jump distribution at every time instant $k$ has a bounded support. A natural question arises: is it possible to construct an ARMA (or AR) type process with marginal distributions of bounded support? This issue is addressed in a number of recent works (see, e.g., [20-23], and references therein). Namely, in [20] the author uses a special random coefficient autoregression to model a first-order AR process with beta marginal distributions. In [21], the authors extend on the earlier works $[22,23]$ and present a first-order autoregressive time-series model with the uniform $(0,1)$ marginal distributions. Autoregressive time-series processes with marginal distributions of other types (not necessarily with bounded supports) have been studies extensively in the literature. Namely, [24] discusses non-Gaussian ARMA processes with marginal distributions of the Laplace and 1-Laplace types. The first-order autoregressive models producing time series with logistic, hyperbolic secant, exponential, Laplace, and Gamma marginal distributions are considered in [25]. In [26], by means of special choice of noise, autoregressive processes with Student type marginal distributions are constructed. An extensive survey of non-Gaussian conditional linear autoregressive models can be found in [27].

\section{Numerical results}

\subsection{Market environment and option characteristics}

In order to test the algorithm under varying market conditions and with a range of option characteristics, data were collected for 55 call options traded on the New York Stock Exchange. The volatility index, or VIX, was used as an indicator of the market environment. The volatility index for a period from September 2002 through April 2007 is shown in Figure 6. Two time periods were selected with differing VIX characteristics. The selected time periods are indicated by the darker sections of the graph between the dashed vertical lines. The first period encompasses dates ranging from October 2002 through October 2004. As shown in the figure, the volatility index was high and widely varying at the beginning of the 


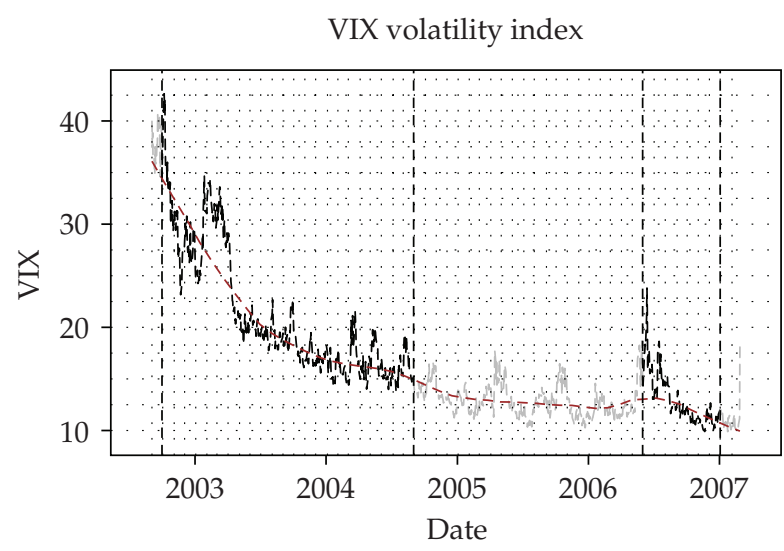

Figure 6: Volatility index.

time period, with lower values towards the end of the period. The second chosen time period was July 2006 through March 2007, with smaller and less variable volatility index values.

Option data was collected for options in the money, at the money and out of the money, from varying industrial sectors and time to expiration. Option characteristics are detailed in Table 4. The data collected for each option was strike price and expiration date, historical stock price data, and historical option prices. Over 500 daily stock prices were recorded. To capture the market behavior corresponding to the volatility index fluctuations occurring at the beginning of 2003, all 500 data values were used in constructing time-series models for the stock price jump process for the options expiring on October 15, 2004. We used 150 historical stock prices in constructing time-series models for the stock price jump process for the options expiring on March 17, 2007 since the volatility index was lower and more stable in this regime. Tests using larger amounts of historical data produced similar results, indicating that a large amount of historical data was not required.

For all options in this study, 100 stock price paths were simulated. The market option price data for a period of 40 days prior to expiration were collected, and the market option price $n$ days prior to expiration was used in identifying the appropriate contour for each of the reported values of $n$ (as described in Section 6).

\subsection{Discussion of algorithm results}

The risk criteria values given in (7.1) through (7.3) were evaluated for each $(u, d)$ pair on the market calibrated contour for each option listed in Table 4 . Table 1 presents the optimal risk criteria values for all options with $n=30$ days to expiration. In addition, the minimum value for each risk criterion is presented in order to gauge the magnitude of the investor's gain associated with choosing the optimal hedging strategy produced by the algorithm. Note that the number at the end of each option ticker indicates the number of days to expiration.

Considering the first option in Table $1(\mathrm{BAC}+\mathrm{CJ} 30)$, we see that the optimal value of $E\left(\Delta_{n}\right)$ or the expected accumulated residual is 0.3446 as reported in column 3 (Residmax), which is over twice as large as the smallest possible value of 0.1701 reported in column 2 (Resid-min). Similarly, we can examine the improvement in shortfall associated with the algorithmic results. The minimum expected shortfall is -0.0226 (Shortfall-min), approximately $10 \%$ of the worst case scenario with a shortfall of -0.2167 (Shortfall-max). The 
Table 1: Algorithmic results for options with 30 days to expiration.

\begin{tabular}{|c|c|c|c|c|c|c|}
\hline & Resid-min & Resid-max & Shortfall-min & Shortfall-max & PosProb-min & PosProb-max \\
\hline $\mathrm{BAC}+\mathrm{CJ} 30$ & 0.1701 & 0.3446 & -0.2167 & -0.0226 & 0.71 & 1.00 \\
\hline $\mathrm{BAC}+\mathrm{CW} 30$ & 0.2446 & 0.3592 & -0.0952 & -0.0035 & 0.98 & 1.00 \\
\hline $\mathrm{BAC}+\mathrm{C} \times 30$ & 0.0776 & 0.3253 & -0.3301 & -0.0865 & 0.52 & 0.87 \\
\hline $\mathrm{BAC}+\mathrm{JV} 30$ & 0.1368 & 0.4406 & -0.4111 & -0.0425 & 0.74 & 0.98 \\
\hline CYQ+CE30 & 0.0301 & 0.5328 & -0.2833 & -0.1226 & 0.60 & 0.97 \\
\hline CYQ+CX30 & 0.1280 & 0.2995 & -0.1182 & -0.0301 & 0.83 & 1.00 \\
\hline CYQ+CY30 & -0.0750 & 1.0162 & -0.5349 & -0.2480 & 0.55 & 0.86 \\
\hline $\mathrm{GE}+\mathrm{CF} 30$ & 0.1542 & 0.1778 & -0.0308 & -0.0004 & 1.00 & 1.00 \\
\hline $\mathrm{GE}+\mathrm{CG} 30$ & 0.0959 & 0.1862 & -0.2934 & -0.0441 & 0.60 & 0.94 \\
\hline $\mathrm{GE}+\mathrm{CY} 30$ & 0.1482 & 0.1776 & -0.0073 & 0.0000 & 1.00 & 1.00 \\
\hline $\mathrm{GE}+\mathrm{CZ} 30$ & 0.0799 & 0.1805 & -0.0964 & -0.0049 & 0.92 & 1.00 \\
\hline GE+JF30 & 0.0259 & 0.0600 & -0.1824 & -0.0159 & 0.35 & 0.95 \\
\hline $\mathrm{HNZ}+\mathrm{CI} 30$ & 0.3118 & 0.6032 & -0.2572 & -0.0166 & 0.95 & 1.00 \\
\hline HNZ+CJ30 & 0.1243 & 0.6220 & -0.3183 & -0.0442 & 0.77 & 1.00 \\
\hline INTC+JE30 & -0.3530 & 0.0502 & -0.3145 & -0.0344 & 0.06 & 0.83 \\
\hline $\mathrm{MER}+\mathrm{CP} 30$ & 0.0945 & 0.3211 & -0.2597 & -0.0101 & 0.85 & 1.00 \\
\hline $\mathrm{MQF}+\mathrm{JX} 30$ & 0.1665 & 0.1921 & -0.0504 & -0.0020 & 0.99 & 1.00 \\
\hline $\mathrm{MS}+\mathrm{CO} 30$ & 0.4038 & 0.7941 & -0.4197 & -0.0319 & 0.89 & 1.00 \\
\hline MSQ+CK30 & 0.0266 & 0.5451 & -0.2825 & -0.0419 & 0.45 & 1.00 \\
\hline MSQ+CY30 & 0.0062 & 0.1225 & -0.0918 & -0.0072 & 0.55 & 1.00 \\
\hline MSQ+JE30 & 0.0915 & 0.1234 & -0.1689 & -0.0201 & 0.69 & 0.98 \\
\hline NQ+CD30 & -0.1216 & 0.1762 & -0.3961 & -0.0885 & 0.18 & 0.93 \\
\hline $\mathrm{WMT}+\mathrm{CH} 30$ & 0.2945 & 0.3740 & -0.1248 & -0.0019 & 1.00 & 1.00 \\
\hline WMT+CJ30 & -0.0872 & 0.0787 & -0.3912 & -0.0777 & 0.29 & 0.84 \\
\hline WMT+JJ30 & -0.1892 & 0.2226 & -0.4898 & -0.0514 & 0.54 & 0.90 \\
\hline $\mathrm{XOM}+\mathrm{CN} 30$ & 0.0058 & 0.5323 & -0.5517 & -0.0542 & 0.31 & 0.98 \\
\hline $\mathrm{XOM}+\mathrm{CO} 30$ & -0.0748 & 1.2703 & -0.9711 & -0.1213 & 0.35 & 0.89 \\
\hline XOM+JI30 & -0.1373 & 0.1527 & -0.2978 & -0.0545 & 0.15 & 0.93 \\
\hline XOM+JV30 & 0.0528 & 0.2231 & -0.1508 & -0.0117 & 0.59 & 1.00 \\
\hline
\end{tabular}

probability of a positive accumulated residual increases from a possible low of 0.71 (PosProbmin) presented in column 6 to 1 (PosProb-max) using the optimal portfolio identified by the algorithm.

In most cases, the expected accumulated residual value is improved by a factor between 1.5 and 3 when the optimal hedging portfolio is chosen. Several cases produced much more dramatic improvements in the objective value. The most significant improvement in expected accumulated residual is found in the results for the XOM+CN30 option. The value associated with the optimal hedging portfolio is 0.5323 , almost 100 times as large as the smallest possible value of .0058 . Other notable cases include the Microsoft options MSQ+CK30 and MSQ+CY30 where the optimal accumulated residual is approximately 20 times as large as the smallest possible value and the Cisco option CYQ+CY30 where the optimal is more than 13 times as large as the smallest possible value.

On average, the shortfall is reduced to approximately $13 \%$ of the largest possible shortfall by following the hedging strategy associated with the optimal hedging portfolio. While the shortfall is reduced to just over 1\% of the worst case for the GE option GE+CF30, 
Table 2: Algorithmic results for options with varying time to expiration.

\begin{tabular}{lccc}
\hline & Resid-max & Shortfall-min & PosProb-max \\
\hline BAC+JV10 & 0.3202 & -0.0085 & 1.00 \\
BAC+JV15 & 0.3044 & -0.0260 & 0.99 \\
BAC+JV20 & 0.2881 & -0.0188 & 1.00 \\
BAC+JV25 & 0.1876 & -0.0334 & 0.99 \\
BAC+JV30 & 0.4406 & -0.0425 & 0.98 \\
GE+CF10 & 0.1302 & 0.0000 & 1.00 \\
GE+CF15 & 0.1612 & 0.0000 & 1.00 \\
GE+CF20 & 0.0615 & -0.0001 & 1.00 \\
GE+CF25 & 0.1179 & -0.0006 & 1.00 \\
GE+CF30 & 0.1778 & -0.0004 & 1.00 \\
\hline
\end{tabular}

the optimal is approximately $46 \%$ of the worst case for the Cisco option CYQ+CY30. It is important to note that the hedging portfolio associated with minimizing shortfall is not the same portfolio choice for maximizing expected accumulated residual.

The Intel option INTC+JE30 provides an interesting example for the benefit of choosing the hedging strategy produced by the algorithm to maximize the probability of a positive accumulated residual. The objective value associated with the optimal strategy is 0.85 as seen in column 7 (ProbPos-max) as compared to values as low as 0.06 (PosProb-min) associated with other strategies.

To investigate the implications of a shorter time horizon, results are summarized for two options (BAC+JV and GE+CF) in Table 2 for $n=30,20,15,10$ days to expiration. The number of days to expiration is indicated by the value at the end of the option ticker in the first column. Examining the first row of the table, we see that for the option BAC+JV with ten days to expiration, the expected accumulated residual has optimal value associated with algorithmic hedging portfolio of 0.3202 (Resid-max), minimum shortfall of -0.0085 (Shortfall$\mathrm{min}$ ), and the probability of a positive accumulated residual of 1.0 (PosProb-max). Following the hedging strategy associated with the optimal choice of $(u, d)$ chosen by the algorithm still provides some advantage as compared to other possible hedging portfolios, although not as much as with a longer time to expiration, as would be expected. The largest improvement in expected accumulated residual for less than 30 days to expiration occurs with the BAC $+\mathrm{JV}$ option with 15 days to expiration. Choosing the optimal hedging strategy provides a value 1.4 times as large as the smallest possible expected accumulated residual. For all cases presented, the minimum shortfall produced by the algorithm is very close to zero and the probability of a positive accumulated residual is very close to 1 .

\section{Evaluation of numerical results}

The results given in Section 9 document the advantages of using the optimal hedging strategy in comparison to choosing from the range of other market calibrated hedging strategies. To further evaluate the results of our risk minimization algorithm, we take the position of an investor possessing a hedging portfolio based on the optimal strategy identified by the algorithm and compute the accumulated residual value using a representative actual stock price path. To obtain representative stock price values, we divide the collected stock price data into two sets: a large set of historical values used to compute the optimal strategy and a 
Table 3: Model evaluation results.

\begin{tabular}{|c|c|c|}
\hline Option & AHR & BSAHR \\
\hline $\mathrm{BAC}+\mathrm{CJ}$ & -0.199 & -0.137 \\
\hline $\mathrm{BAC}+\mathrm{CW}$ & 0.354 & 0.278 \\
\hline $\mathrm{BAC}+\mathrm{CX}$ & -1.977 & -0.444 \\
\hline $\mathrm{BAC}+\mathrm{JV}$ & 0.476 & 0.177 \\
\hline $\mathrm{CYQ}+\mathrm{CE}$ & 0.557 & 0.282 \\
\hline CYQ+CX & 0.30 & 0.281 \\
\hline $\mathrm{CYQ}+\mathrm{CY}$ & 0.016 & 0.186 \\
\hline $\mathrm{GE}+\mathrm{CG}$ & 0.438 & 0.102 \\
\hline $\mathrm{GE}+\mathrm{CY}$ & 0.202 & 0.170 \\
\hline $\mathrm{GE}+\mathrm{CZ}$ & 0.180 & 0.168 \\
\hline $\mathrm{GE}+\mathrm{JF}$ & 0.075 & 0.066 \\
\hline $\mathrm{HNZ}+\mathrm{CI}$ & 0.590 & 0.053 \\
\hline $\mathrm{HNZ}+\mathrm{CJ}$ & -0.283 & 0.144 \\
\hline INQ+JE & -0.024 & -0.073 \\
\hline $\mathrm{INQ}+\mathrm{CD}$ & -0.513 & 0.059 \\
\hline $\mathrm{MER}+\mathrm{CP}$ & 0.044 & 0.254 \\
\hline $\mathrm{MS}+\mathrm{CO}$ & -0.433 & -0.097 \\
\hline $\mathrm{MQF}+\mathrm{JX}$ & 0.185 & 0.180 \\
\hline MSQ+CK & -1.793 & -0.101 \\
\hline MSQ+JE & 0.132 & 0.123 \\
\hline $\mathrm{WMT}+\mathrm{CH}$ & 0.218 & 0.318 \\
\hline $\mathrm{WMT}+\mathrm{CJ}$ & -0.016 & -0.166 \\
\hline WMT+JJ & 0.315 & 0.286 \\
\hline $\mathrm{XOM}+\mathrm{CN}$ & 0.434 & 0.506 \\
\hline $\mathrm{XOM}+\mathrm{CO}$ & -2.90 & -0.034 \\
\hline $\mathrm{XOM}+\mathrm{JI}$ & 0.254 & 0.004 \\
\hline $\mathrm{XOM}+\mathrm{JV}$ & 0.234 & 0.199 \\
\hline
\end{tabular}

AHR: Algorithmic Accumulated Hedge Residual.

BSAHR: Black-Scholes Accumulated Hedge Residual.

set of $n+1$ values, where $n$ is the number of days to expiration of the option, to be interpreted as the actual values of the stock (we use $n=30$ days to expiration for this study).

Figure 7 illustrates this data splitting for a set of Bank of America stock prices from October 15, 2002 through October 15, 2004. To evaluate the optimal hedging portfolio produced by the risk minimization algorithm for an option $n=30$ days to expiration, the stock prices for October 15, 2002 through September 1, 2004 are used in the algorithm to compute the optimal hedging strategy, and stock prices from September 2, 2004 through October 15, 2004 (highlighted in gray) are used as the actual stock price values. The stock price and option price on the first day of the actual stock price path are used as the current stock and option price. The data used as the actual stock path is shown in more detail in the lower graph in Figure 7.

Having computed the accumulated residual for the single actual path, we compare these to the hedging cost of maintaining the classic Black-Scholes hedge when rebalancing is done daily. Thus, in parallel with the computation of our residuals, we compute the accumulated rebalancing cost of the Black-Scholes hedge, as described in [28]. To be consistent with the method described in [28], we account for the future value (value 
Table 4: Options used in data analysis.

\begin{tabular}{lcccc}
\hline Company name & Option & Days to expiration $(n)$ & Strike price & Expiration data \\
\hline Bank of America Corp. & BAC+CJ & 30 & 50 & March 17, 2007 \\
Bank of America Corp. & BAC+CW & 30 & 47.50 & March 17, 2007 \\
Bank of America Corp. & BAC+CX & 30 & 52.50 & March 17, 2007 \\
Bank of America Corp. & BAC+JV & $10,15,20,25,30$ & 42.50 & October 15, 2004 \\
Cisco Systems, Inc. & CYQ+CE & 30 & 25 & March 17, 2007 \\
Cisco Systems, Inc. & CYQ+CX & 30 & 22.50 & March 17, 2007 \\
Cisco Systems, Inc. & CYQ+CY & 30 & 27.50 & March 17, 2007 \\
Exxon Mobil Corp. & XOM+CN & 30 & 70 & March 17, 2007 \\
Exxon Mobil Corp. & XOM+CO & 30 & 75 & March 17, 2007 \\
Exxon Mobil Corp. & XOM+JI & 30,20 & 45 & October 15, 2004 \\
Exxon Mobil Corp. & XOM+JV & $30,20,10$ & 42.5 & October 15, 2004 \\
General Electric Co. & GE+CF & $10,15,20,25,30$ & 30 & March 17, 2007 \\
General Electric Co. & GE+CG & $10,20,30$ & 35 & March 17, 2007 \\
General Electric Co. & GE+CY & 30 & 27.5 & March 17, 2007 \\
General Electric Co. & GE+CZ & 30 & 32.50 & March 17, 2007 \\
General Electric Co. & GE+JF & 30 & 30 & October 15, 2004 \\
HJ Heinz Co. & HNZ+CI & 30 & 45 & March 17, 2007 \\
HJ Heinz Co. & HNZ+CJ & 30 & 50 & March 17, 2007 \\
Intel Corp. & INQ+CD & $30,20,10$ & 20 & March 17, 2007 \\
IntelCorp. & INQ+JE & $30,20,10$ & 25 & October 15, 2004 \\
Merrill Lynch\& Co., Inc. & MER+CP & 30 & 80 & March 17, 2007 \\
Morgan Stanley & MS+CO & 30 & 75 & March 17, 2007 \\
Microsoft Corp. & MSQ+CK & 30 & 30 & March 17, 2007 \\
Microsoft Corp. & MSQ+CY & 20,30 & 27.50 & March 17, 2007 \\
Microsoft Corp. & MSQ+JE & 30 & 25 & October 15, 2004 \\
Microsoft Corp. & MQF+JX & 30 & 50 & October 15, 2004 \\
Walmart Stores, Inc. & WMT+CH & $10,15,20,25,30$ & 50 & March 17, 2007 \\
Walmart Stores, Inc. & WMT+CJ & $10,20,30$ & October 15, 2004 \\
Walmart Stores, Inc. & WMT+JJ & $30,20,7$ & & \\
\hline & & & 5007 \\
\hline
\end{tabular}

at expiration) of the option premium received when we take the position of the option seller. It consists of adding the future value of the option premium to the accumulated residuals at expiration. We call the resulting value the accumulated hedging residual. Positive accumulated hedging residuals indicate a gain for the portfolio holder, while a negative accumulated hedging residual indicates a loss for the portfolio holder.

Accumulated residual for both the optimal hedging strategy produced by the algorithm and the Black-Scholes hedge is presented in Table 3. Of the twenty-seven options listed in the table, there are sixteen for which our accumulated hedge residual exceeds that of the Black-Scholes hedge. The results illustrate that following the hedging portfolio strategy produced by the algorithm is beneficial to an investor, producing residual values that exceed the classic Black-Scholes value in $59 \%$ of the test cases. The number of favorable cases provides support for the use of our algorithm. 


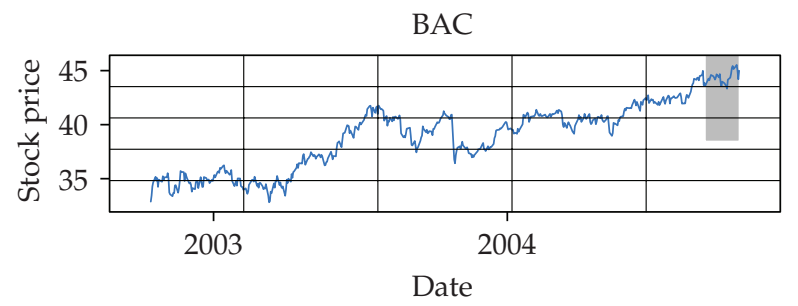

(a)

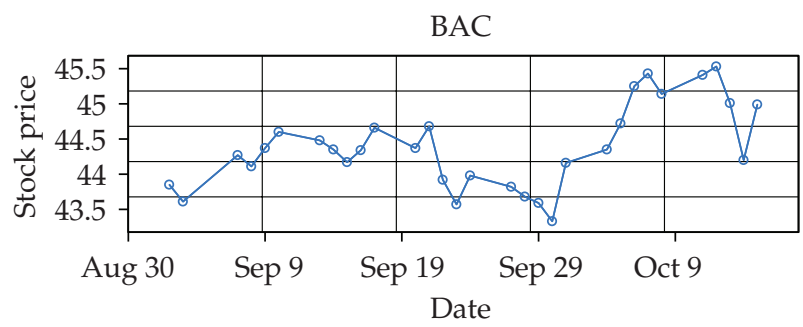

(b)

Figure 7: Stock price data.

\section{Conclusions}

We have developed an algorithm based on an ARMA time-series model for a stock price jump process that produces a non-self-financing hedging strategy in an incomplete market corresponding to one of several investor risk criteria. The two-stage algorithm optimizes an investor chosen statistical property of the portfolio residual profit or shortfall. The algorithm was tested on a number of options traded on the New York Stock Exchange.

\section{Acknowledgments}

The authors would like to thank N. N. Leonenko for his helpful discussions and insight as well as for providing us with important references. They would also like to thank the anonymous referees for their suggestions that have improved the quality of the presentation. This work is partially supported by the Bentley Fund for Strategic Research.

\section{References}

[1] N. Josephy, L. Kimball, V. Steblovskaya, A. V. Nagaev, and M. Pasniewski, “An algorithmic approach to non-self-financing hedging in a discrete-time incomplete market," Discrete Mathematics and Applications, vol. 17, no. 2, pp. 189-207, 2007.

[2] A. V. Nagaev and S. A. Nagaev, "Asymptotics of riskless profit under selling of discrete time call options," Applicationes Mathematicae, vol. 30, no. 2, pp. 173-191, 2003.

[3] S. A. Nagaev, A. V. Nagaev, and R. M. Kunst, "A diffusion approximation to the Markov chains model of the financial market and the expected riskless profit under selling of call and put options," Economics Series 165, Institute for Advanced Studies, Vienna, Austria, 2005.

[4] S. A. Nagaev, A. V. Nagaev, and R. M. Kunst, "A diffusion approximation for the riskless profit under selling of discrete time call options: non-identically distributed jumps," Economics Series 164, Institute for Advanced Studies, Vienna, Austria, 2005.

[5] A. V. Nagaev, "A diffusion approximation of the expected risky profit on an investor under selling of discrete time options" (Russian), working paper. 
[6] G. Tessitore and J. Zabczyk, "Pricing options for multinomial models," Bulletin of the Polish Academy of Sciences, Mathematics, vol. 44, no. 3, pp. 363-380, 1996.

[7] G. Wolczynska, "An explicit formula for option pricing in discrete incomplete markets," International Journal of Theoretical and Applied Finance, vol. 1, no. 2, pp. 283-288, 1998.

[8] O. Hammarlid, "On minimizing risk in incomplete markets option pricing models," International Journal of Theoretical and Applied Finance, vol. 1, no. 2, pp. 227-233, 1998.

[9] J. Staum, "Incomplete markets," in Handbooks in Operations Research and Management Science, vol. 15, pp. 511-564, Elsevier Science, Amsterdam, The Netherlands, 2006.

[10] E. Jouini, J. Cvitanić, and M. Musiela, Eds., Option Pricing, Interest Rates and Risk Management, Handbooks in Mathematical Finance, Cambridge University Press, Cambridge, UK, 2001.

[11] A. N. Shiryaev, Essentials of Stochastic Finance: Facts, Models, Theory, vol. 3 of Advanced Series on Statistical Science \& Applied Probability, World Scientific, River Edge, NJ, USA, 1999.

[12] A. V. Mel'nikov, S. N. Volkov, and M. L. Nechaev, Mathematics of Financial Obligations, vol. 212 of Translations of Mathematical Monographs, American Mathematical Society, Providence, RI, USA, 2002.

[13] V. Ryabchenko, S. Sarykalin, and S. Uryasev, "Pricing European options by numerical replication: quadratic programming with constraints," Asia-Paciffc Financial Markets, vol. 11, no. 3, pp. 301-333, 2004.

[14] W. B. Arthur, J. H. Holland, B. LeBaron, R. Palmer, and P. Tayler, "Asset pricing under endogenous expectations in an artificial stock market," in The Economy as an Evolving, Complex System II, AddisonWesley, Reading, Mass, USA, 1997.

[15] J. Benhabib, Cycles and Chaos in Economic Equilibrium, Princeton University Press, Princeton, NJ, USA, 1992.

[16] W. A. Brock and C. Sayers, "Is the business cycle characterized by deterministic chaos?" Journal of Monetary Economics, vol. 22, no. 1, pp. 71-90, 1988.

[17] C. W. Granger, "Is chaotic economic theory relevant for economics? A review essay," Journal of International and Comparative Economics, vol. 3, pp. 139-145, 1994.

[18] L. Rüschendorf, "On upper and lower prices in discrete-time models," Proceedings of the Steklov Institute of Mathematics, vol. 237, pp. 134-139, 2002.

[19] R. Carmona, Statistical Analysis of Financial Data in S-Plus, Springer Texts in Statistics, Springer, New York, NY, USA, 2004.

[20] E. McKenzie, “An autoregressive process for beta random variables," Management Science, vol. 31, no. 8, pp. 988-997, 1985.

[21] M. Ristić and B. Popović, "A new uniform AR(1) time series model (NUAR(1))," Publications de l'Institut Mathématique, vol. 68(82), pp. 145-152, 2000.

[22] M. R. Chernick, "A limit theorem for the maximum of autoregressive processes with uniform marginal distributions," The Annals of Probability, vol. 9, no. 1, pp. 145-149, 1981.

[23] A. J. Lawrance, "Uniformly distributed first-order autoregressive time series models and multiplicative congruential random number generators," Journal of Applied Probability, vol. 29, no. 4, pp. 896-903, 1992.

[24] L. S. Dewald Sr., P. A. W. Lewis, and E. McKenzie, "Marginally specific alternatives to normal ARMA processes," in Proceedings of the 19th Winter Simulation Conference (WSC '87), A. Thesen, H. Grant, and W. D. Kelton, Eds., pp. 300-301, Atlanta, Ga, USA, December 1987.

[25] C. H. Sim, "Modelling non-normal first-order autoregressive time series," Journal of Forcasting, vol. 13, no. 4, pp. 369-381, 1994.

[26] C. C. Heyde and N. N. Leonenko, "Student processes," Advances in Applied Probability, vol. 37, no. 2, pp. 342-365, 2005.

[27] G. K. Grunwald, R. J. Hyndman, L. Tedesco, and R. L. Tweedie, "Non-Gaussian conditional linear AR(1) models," Australian \& New Zealand Journal of Statistics, vol. 42, no. 4, pp. 479-495, 2000.

[28] J. C. Hull, Options, Futures and Other Derivatives, Prentice Hall, Upper Saddle River, NJ, USA, 6th edition, 2005. 


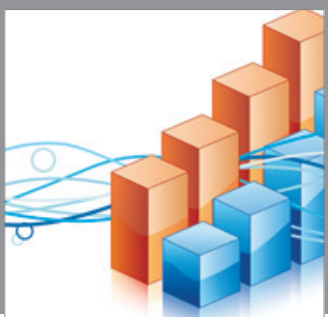

Advances in

Operations Research

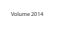

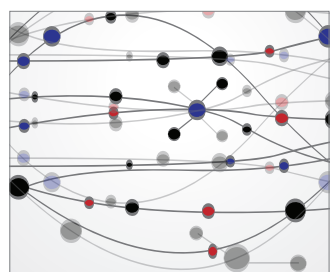

\section{The Scientific} World Journal
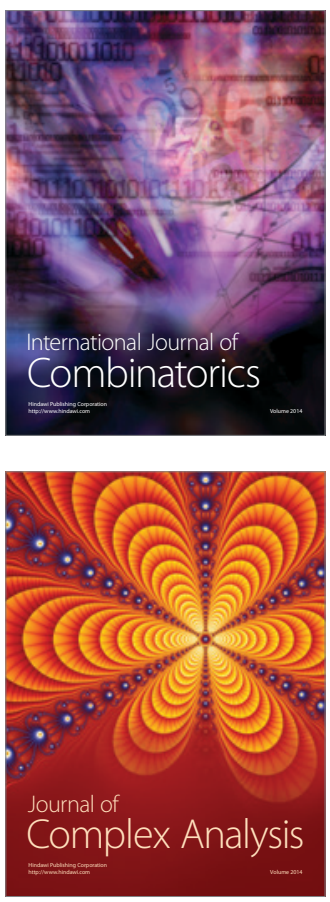

International Journal of

Mathematics and

Mathematical

Sciences
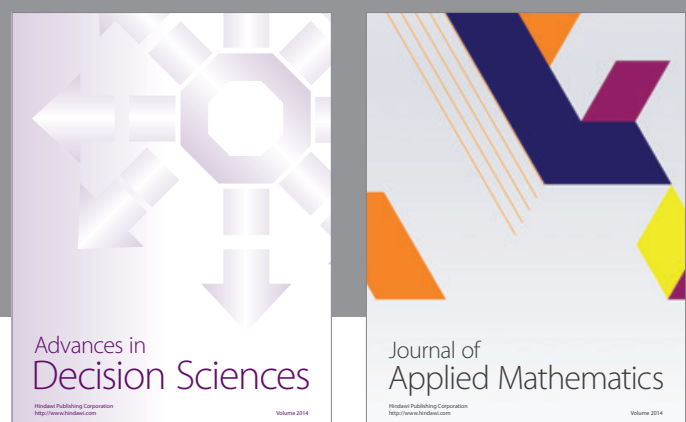

Journal of

Applied Mathematics
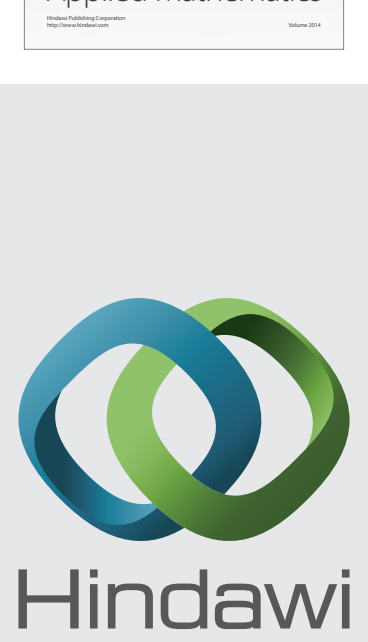

Submit your manuscripts at http://www.hindawi.com
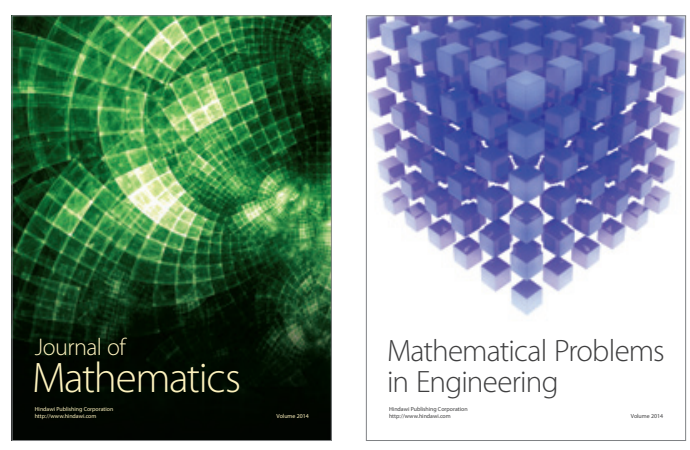

Mathematical Problems in Engineering
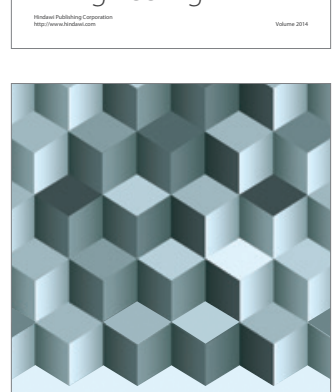

Journal of

Function Spaces
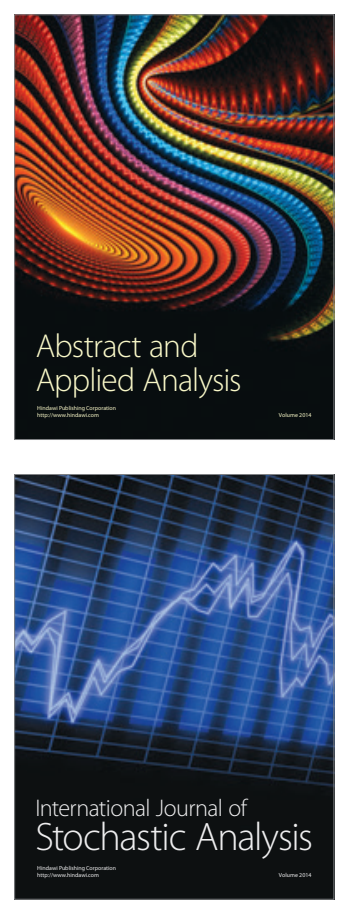

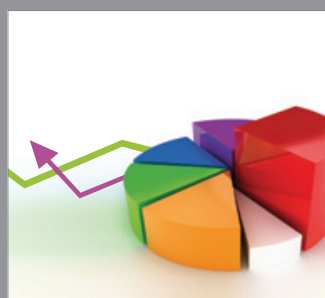

ournal of

Probability and Statistics

Promensencen
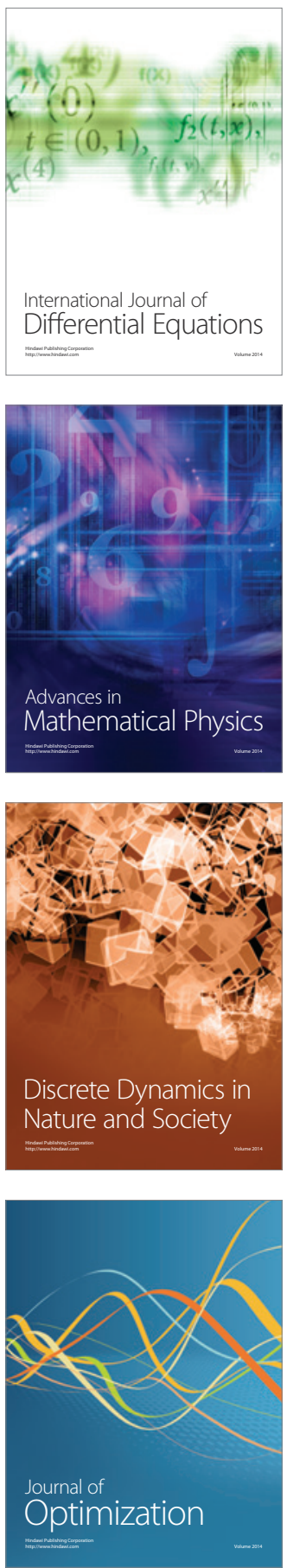